\title{
Le travail parental : du côté des pères séparés et divorcés
}

\section{Agnès Martial, anthropologue}

L'arrivée, annoncée ici et là, des "nouveaux pères », investis et valorisés dans les soins domestiques et éducatifs aux enfants, est-elle observable dans la réalité ? Une revue de la littérature scientifique récente sur le sujet nous indique qu'elle l'est en partie parmi les pères séparés et divorcés. Ce que la situation de ces derniers a de spécifique nous éclaire en creux sur la notion de travail parental, sur les pères et mères d'aujourd'hui et sur la persistance de modèles et de pratiques « genrées » différenciés.

La notion de travail parental apparaît comme un fécond révélateur de l'évolution des statuts et des rôles sexués au sein du couple et de la famille. Recouvrant les différents gestes, savoir-faire et compétences liés à l'accomplissement des soins et des tâches relatifs à l'éducation quotidienne des enfants, elle requalifie des actes traditionnellement attribués à la sphère féminine. Les termes évoquant un « travail », un « métier » et des « carrières » associés aux fonctions parentales sont issus du monde professionnel, univers de sociabilité qui fut traditionnellement celui des hommes entre eux, et lieu privilégié de la construction des identités masculines. L'égalitarisation des rôles de genre est, en outre, plus rapide dans la sphère professionnelle que dans la sphère familiale. L'idée d'un travail parental tend donc à neutraliser des tâches, des attitudes et des fonctions longtemps associées à la maternité, et dont on attend désormais qu'elles soient également investies par les hommes. Les normes psychosociales encouragent en effet ces derniers, au nom d'un partage plus égalitaire, à investir une relation paternelle « nourricière », quotidienne, et plus individualisée (Neyrand, 2000 ; Castelain-Meunier, 2002 ; Bergonnier-Dupuy et Robin, 2007). Le concept de travail parental se fait ainsi l'écho d'un modèle social et juridique qui promeut, en France comme dans la majeure partie des pays occidentaux, l'égalité et la coparentalité. Mais le risque est alors d'occulter la réalité des asymétries présentes dans l'accomplissement des tâches et des rôles parentaux.

\section{L'asymétrie du travail parental dans les situations de séparation}


La rupture conjugale et l'organisation post-séparation de la prise en charge des enfants sont des événements particulièrement révélateurs de ces asymétries. De nombreux travaux portant sur le divorce et sur les familles recomposées soulignent ainsi le maintien d'une répartition des rôles et des tâches qui laisse à la mère la plus grande part des responsabilités et des contraintes parentales, si bien que les premières recherches françaises portant sur les rapports de genre au sein des organisations familiales succédant à la rupture évoquaient une forme de « monoparentalité éducative » produite par une forte tendance à la « matricentralité » (Blöss, 1996 ; Cadolle, 2000). Ainsi le travail parental repose-t-il principalement et majoritairement sur des mères assumant la plus grande partie des tâches éducatives et domestiques dans les configurations familiales issues des divorces et des séparations. De manière symétrique, le statut et le rôle des pères séparés ou divorcés ont, le plus souvent, été analysés en référence aux notions de retrait, d'éloignement, d'absence, de défaillance et de non-investissement dans la relation aux enfants.

Il est vrai que dans un contexte où les enfants vivent au domicile maternel dans neuf cas sur dix (Bonvalet et Lelièvre, 2006), le droit de visite et d'hébergement attribué aux pères ou demandé par eux (1) offre un espace limité à l'accomplissement du travail parental, dès lors qu'il circonscrit ou empêche l'expérience de la quotidienneté et de la corésidence (Martial, 2003). Discontinue, enclose dans un temps réduit et soumise à l'appréciation positive des visites par l'enfant, la relation paternelle devient vouée à des activités récréatives orchestrées par des «Disneyland dads » (Stewards, 1999) qui laissent à la mère l'essentiel de la charge pratique et mentale du travail parental.

Pourtant, et même si l'on connaît la tendance à la fragilisation des liens père/enfant dans ces situations (2), divorces et séparations sont susceptibles d'offrir un lieu fécond pour l'analyse du travail parental tel qu'il peut être accompli par les pères, parce qu'ils créent des situations où les hommes se trouvent en position d'assumer leur rôle de parent en l'absence de la mère. Se développent, par exemple, des situations de résidence alternée qui instaurent un partage égal du temps quotidien de l'enfant entre son père et sa mère. Il arrive également que des hommes séparés ou divorcés deviennent pères « gardiens », assumant intégralement l'éducation de leurs enfants. Les cas évoqués ici reflètent bien sûr une minorité de situations : les pères (veufs, séparés, divorcés) qui élèvent seuls leurs enfants représentaient, en 1999, $14 \%$ des familles monoparentales, et $2,5 \%$ de l'ensemble des familles avec enfants de moins de 25 ans (Eydoux et Letablier, 2007). La proportion d'enfants vivant en résidence alternée suite à la séparation de leurs parents demeure, quant à elle, tout aussi limitée : cette situation ne concerne guère plus qu'un dixième des familles (3). Ces cas n'en sont pas moins « exemplaires », au sens où ils permettent d'explorer, à partir de 
situations inédites et innovantes, les enjeux d'une question sociale dont ils ébranlent le cadre ordinaire et les apparentes évidences.

Dans ces différents cas de paternité quotidienne et « solitaire », qu'elle soit en alternance ou en continu, de quelle manière le travail parental engage-t-il la définition des hommes comme pères ? Quelles sont les modalités de la redéfinition des statuts parentaux ? Comment ces hommes perçoivent-ils, entre paternité et masculinité, leur statut et leur identité sexuée?

Pour apporter quelques éléments de réponse à ces questions, nous nous appuierons sur des travaux empiriques analysant, dans ces situations, les enjeux relatifs au travail parental.

\section{Le travail parental : des différences selon le genre ?}

La définition du travail parental comporte une dimension variable et fluctuante. Il peut faire l'objet d'interprétations différentes, et l'accomplissement par les pères et les mères de tâches apparemment similaires ne conduit pas nécessairement à l'équivalence des investissements parentaux. Certaines études portant à la fois sur des pères séparés et sur des pères au foyer vivant en couple qui partagent tout ou partie du quotidien avec les enfants dont ils prennent soin pointent ainsi la persistance des modèles présidant à la définition des rôles « genrés » dans l'accomplissement du travail parental. Andréa Doucet (2006) montre, par exemple, à partir d'une enquête menée au Canada sur les pères au foyer et les pères séparés ou divorcés, que le travail parental au quotidien les amène à intégrer dans leur paternité des tâches traditionnellement définies comme féminines, mais qu'il ne conduit pas à l'indistinction des rôles paternels et maternels. Même quand les pères fournissent les soins quotidiens à leurs enfants, ils évoquent des différences fondamentales entre « maternage » et « paternage », bien que les gestes accomplis ne semblent pas si différents de ceux des mères. De plus, certains présentent moins leur investissement comme une nouvelle facette de leur paternité que comme un substitut au travail maternel palliant l'absence de la conjointe ou de l'ex-conjointe : le travail parental qu'ils accomplissent ne transforme donc pas leur définition de la paternité. Ils minimisent la signification des responsabilités mises en jeu, et présentent finalement leur relation aux enfants comme basée sur le plaisir et le jeu, et isolée du reste du travail parental. De manière réciproque, dans les couples où les pères sont au foyer, les femmes qui occupent le rôle de pourvoyeuse n'abandonnent pas toujours la responsabilité et la charge mentale relatives à la vie domestique, et continuent en réalité de gérer une multiplicité de contraintes familiales et professionnelles. Chacun réfère alors très différemment au travail parental pour se définir comme parent, et les clivages traditionnels persistent en dépit de la nouvelle organisation familiale...

Les travaux récents menés en France sur les usages associés à la résidence alternée témoignent également d'interprétations et 
d'investissements différenciés de la prise en charge quotidienne des enfants, qui peut devenir un fort enjeu de conflit entre les parents. Sylvie Cadolle, à partir d'une enquête menée auprès d'une vingtaine d'hommes et de femmes ayant opté pour le système de la résidence alternée (4), oppose ainsi le sentiment de satisfaction des pères pratiquant ce système et les contrariétés exprimées par certaines mères, qui doutent des compétences éducatives de leur ex-conjoint et leur reprochent leur indisponibilité à l'égard des enfants.

Rappelons que le travail parental relève traditionnellement du domaine des femmes, qu'il est effectué majoritairement par les mères, et par conséquent défini par elles et selon leurs critères. Ceci peut éclairer le discours disqualifiant de certaines des mères rencontrées par la sociologue quant aux capacités éducatives de leur ex-conjoint. Les mères qui font confiance au père de leurs enfants sont celles qui ont vécu avec lui au sein de couples décrits comme égalitaires, où les tâches relatives au travail parental étaient partagées dès avant la séparation, celui-ci ne relevant plus d'un domaine spécifiquement maternel déterminant les critères de "bonnes pratiques" éducatives. Notons que dans ces couples, les femmes étaient par ailleurs autant voire plus investies professionnellement que leur conjoint.

\section{Travail parental et rapport à l'emploi}

La question du rapport à l'emploi est évidemment centrale dans les réajustements relatifs au partage du travail parental, pour les parents séparés comme pour ceux vivant en couple. Sylvie Cadolle met ainsi en évidence l'asymétrie des modes de conciliation entre vie familiale et vie professionnelle dans certaines situations de résidence alternée, les inégalités de temps investi par chacun des ex-conjoints se trouvant reconduites après la séparation. De là proviennent d'importants conflits mais aussi, dans les couples qu'elle qualifie de « traditionnels rénovés », des arrangements par lesquels les hommes demeurent plus investis professionnellement que leur ex-conjointe, celle-ci se rendant plus disponible pour leurs enfants. L'alternance de la résidence n'est alors pas équivalente en nombre de jours, mais elle permet, dans les moments où les pères peuvent accueillir leurs enfants, un partage du temps quotidien plus important entre père et enfant que dans I'organisation classique du « droit de visite et d'hébergement ».

Ces différents résultats soulignent la force, même au sein d'organisations conduisant à la prise en charge, par les pères, de tout ou partie du quotidien des enfants, de l'assignation des femmes à la gestion de la sphère familiale et domestique et de celle des hommes à la sphère professionnelle. La figure du père pourvoyeur demeure fortement présente dans la construction des identités masculines (Connell, 1999 ; Merla, 2007) et limite l'investissement des pères séparés et divorcés dans l'accomplissement du travail parental au quotidien. 
Des situations minoritaires témoignent cependant de nouvelles formes de conciliation entre vie familiale et vie professionnelle chez certains pères séparés ou divorcés. Terry Arrendel (1995) le constatait aux Etats-Unis, dès le début des années 1990, décrivant, pour un tout petit nombre de pères (neuf hommes sur les soixantequinze pères séparés ou divorcés interviewés), une érosion de l'adhésion à une définition « conventionnelle » de la masculinité associée à la réussite professionnelle. À la suite de leur divorce, dans leur nouvelle situation familiale, ces pères avaient opéré des choix privilégiant une présence plus importante au foyer, supposant le renoncement - au moins provisoire - à une carrière ascendante, un changement d'emploi impliquant parfois une baisse de revenu et, de manière générale, un renversement de l'ordre des priorités familiale et professionnelle parfois difficile à mettre en œuvre.

Dans certains cas, l'investissement d'un rôle parental au quotidien peut même offrir aux hommes un statut, une identité sociale que ne fournit plus le travail salarié. Une étude réalisée au Québec sur des pères séparés, en situation de grande précarité, ayant maintenu leur « engagement paternel » met en évidence l'atténuation de la dimension du rôle de pourvoyeur chez des pères qui ne peuvent recourir qu'à des emplois précaires aux horaires flexibles, irréguliers, atypiques et difficilement conciliables avec leur rôle parental. L'exclusion du marché du travail devient pour ces hommes un « sacrifice nécessaire », au moins pour un temps, au fait d'assumer leur responsabilité parentale. Dans un contexte de fragilisation du rapport à l'emploi, le travail parental est devenu pour certains de ces pères un élément plus valorisant que le statut professionnel, l'enfant « devenant la composante la plus gratifiante et centrale de leur vie et leur conférant du même coup un statut social » (Allard et al., 2005 , p. 25). Ils adoptent, en cela, des attitudes classiquement attribuées aux mères.

\section{Les pères seuls face au quotidien du travail parental}

Ces pères décrivent d'ailleurs avec force détails les soins matériels et affectifs qu'ils donnent à leurs enfants, évoquant à la fois leurs soucis éducatifs et la satisfaction qu'ils éprouvent dans l'accomplissement du travail parental. De manière comparable, certains des pères séparés ou divorcés rencontrés par $\mathrm{T}$. Arrendel décrivent les difficultés qu'ils ont affrontées lorsqu'ils ont dû assumer seuls la prise en charge quotidienne de leurs enfants, et soulignent l'importance de ces gestes pour leur relation à ceux-ci. Nombreux sont les pères décrivant la mise en place de routines composées des actes nourriciers, ménagers et éducatifs de la vie domestique et familiale. Ces activités, réalisées au quotidien ou durant le temps de visite de l'enfant, concernent les jeux mais aussi les courses et la préparation de repas, l'aide aux devoirs et le suivi scolaire, matérialisant une présence affective et éducative. Ainsi le travail parental conduit-il à 
maintenir, voire à redéfinir et à renforcer, la relation père/enfant au quotidien dans le contexte des séparations.

Dans certains cas enfin, les pères assument seuls ou presque l'éducation de leurs enfants, dès lors que la mère est absente ou peu présente dans la vie de ces derniers. Le récit de leur paternité tel que l'a recueilli T. Arrendel présente alors de fortes similitudes avec l'expérience des mères de familles monoparentales. La charge mentale, physique et financière que représente la paternité « en solo », la nécessité d'être disponible à tout moment et de répondre à tous les besoins d'un enfant (tant du point de vue de la gestion du quotidien que du suivi scolaire, des loisirs ou encore des ressources affectives), la multiplicité des contraintes familiales et professionnelles, ainsi que l'importance du soutien de l'ex-conjoint et des grands-parents sont évoques par ces pères. Ces hommes expriment aussi un fort sentiment de solitude, sans doute aggravé par l'absence de modèle, de valorisation et de soutien pour ces figures paternelles singulières.

\section{La transgression des normes de genre}

La dimension sexuée du travail parental amène en effet les hommes qui se trouvent en situation de l'accomplir au quotidien à transgresser l'existence d'espaces et de réseaux sociaux « genrés » où les pères peinent à trouver place. Laura Merla (2007) souligne ainsi, au sujet des pères au foyer en Belgique, les difficultés que doivent affronter les hommes lorsqu'ils s'approprient les gestes et les pratiques du soin aux enfants, investissant alors des espace sociaux massivement féminins (parcs, aires de jeux, spectacles pour enfants...), ou attribués à la sphère masculine et non adaptés à la présence de jeunes enfants.

Divers auteurs évoquent aussi la remise en question, par l'entourage proche et l'environnement social, de la conformité de ces pères aux normes définissant la masculinité (virilité, force physique et hétérosexualité). T. Arrendel qualifie d'ailleurs les hommes divorcés investis dans le travail parental auprès de leurs enfants de pères « androgynes », parce qu'ils disent s'être approprié des comportements parentaux et des attitudes caractérisés comme féminins, évoquant notamment l'importance des soins nourriciers aux enfants, et référant à des modèles féminins dans leur environnement proche (la mère, l'ex-conjointe ou la sœur). Ces pères expérimentent d'importants conflits intra-genre, affrontant un discours réflexif négatif sur leur manière d'être et de faire avec leurs enfants, notamment de la part de l'entourage masculin. Ce manque de reconnaissance peut conduire à un sentiment d'exclusion et d'isolement à l'égard des formes de sociabilité des deux sexes, lié à la fois à la difficulté d'accéder aux réseaux féminins constitués autour de l'enfance et à l'absence d'un groupe de pairs masculins partageant la même expérience. Pascale Jamoulle (2004), dans son enquête sur la paternité en milieu précaire, évoque ainsi les 
sentiments d'incompétence, de non-reconnaissance, et l'immense solitude des quelques pères gardiens qu'elle a rencontrés, dans un contexte socioculturel où les rôles et les espaces sociaux de la parentalité demeurent fortement sexués.

Ici encore, l'étude menée au Québec sur les pères demeurés engagés auprès de leurs enfants en contexte de pauvreté apporte un éclairage intéressant. Ces pères ont redéfini leur rôle dans l'espace privé, ils montrent une forte assurance dans leurs compétences parentales qu'ils décrivent en détail et ne semblent pas accorder d'importance au genre dans la définition des rôles parentaux. Or, l'enquête souligne la présence constante, dans leur cheminement, d'un réseau de soutien familial et social les encourageant à continuer d'assumer leurs responsabilités parentales, et leur capacité à faire appel à diverses aides institutionnelles, à travers le recours à des services sociaux habituellement sollicités et fréquentés par des femmes.

Les formes, les usages et les significations du travail parental accompli par les pères séparés ou divorcés ouvrent de riches horizons de recherche. On y observe comme ailleurs l'apparition d'un modèle de père nourricier, investi dans les soins domestiques et éducatifs aux enfants et valorisé dans cette fonction. Mais la réalité de cette figure parentale demeure circonscrite à une minorité de cas. Tout d'abord, le recours au système du droit de visite et $\mathrm{d}$ 'hébergement des enfants par leur père limite les possibilités d'investissement parental au quotidien. De plus, la paternité « au jour le jour » ne donne pas toujours lieu à une véritable redéfinition des rôles et des tâches, reconduisant parfois, dans un nouveau contexte organisationnel, les arrangements de genre traditionnels. Enfin, la question du rapport à l'emploi joue un rôle majeur : la répartition, la signification et la valeur du travail parental ne prennent sens qu'au regard de la redéfinition du rapport des pères et des mères à la sphère professionnelle. Le travail parental n'en apparaît pas moins comme l'une des dimensions essentielles du contenu et du maintien des relations père/enfant après une rupture d'union. On y voit se profiler la redéfinition d'une identité masculine transgressant des frontières de genre encore tenaces, et l'importance, au-delà des principes proclamés, du regard et de la reconnaissance sociale et institutionnelle accordés à ces nouvelles formes de paternité.

Notes

1 - Dans les années 1990, les demandes de résidence de l'enfant chez le père étaient de moins de deux sur dix (Neyrand, 2000), la « garde à la mère » étant par ailleurs majoritairement instaurée dans les divorces par consentement mutuel, où les arrangements résidentiels étaient choisis par les parents et entérinés par le juge 
(Théry, 1993). La loi du 4 mars 2002 instaurant le partage égal de l'autorité parentale entre les parents séparés augmente, pour les pères qui le demandent, les chances d'obtenir la résidence de l'enfant.

2 - En 1994, 15 \% de l'ensemble des enfants, en France, ne vivaient pas avec leur père et, parmi ces enfants, un sur trois ne le voyait jamais (Villeneuve-Gokalp, 1999). Les recherches les plus récentes constatent qu'à l'âge adulte, la séparation ou le divorce des parents fragilise davantage les relations au père (Regnier-Loilier, 2006 ; Vivas, 2007).

3 - « En 2005, la proportion d'enfants faisant l'objet d'une résidence en alternance en vertu d'une décision de justice se situait, tous âges confondus, aux alentours de $11 \%(10,8 \%)$ » (Brunet et al., 2008, p. 15).

4 - Enquête menée dans le cadre du projet de recherche ATIP (Action Incitative Jeune Chercheur) « Les partages au sein des couples. Normes juridiques et usages sociaux de l'argent et des biens », dirigé par Agnès Martial, et dont une partie des résultats a déjà été publiée (Cadolle, 2008).

\section{Bibliographie}

> Allard F., Bourret A., Tremblay G., Bergeron M. et Roy I., 2005, « Maintien de l'engagement paternel après une rupture conjugale : point de vue de pères vivant en contexte de pauvreté », Enfances, familles, générations, $n^{\circ} 3$, «Paternité : bilan et perspective » (www.erudit.org/revue/efg/).

> Arrendell T., 1995, Fathers and Divorce, Newbury Park, California, Sage Publications.

> Blöss T., 1996, Éducation familiale et beau-parenté. L'empreinte des trajectoires biographiques, Paris, L'Harmattan. > Bergonnier-Dupuy G. et Robin M., 2007, Couple conjugal, couple parental : vers de nouveaux modèles, Érès.

$>$ Bonvalet C. et Leliève E., 2006, « Les relations parents/enfants après décohabitation », Note rapide Population-modes de vie, $n^{\circ}$ 376/G, Iaurif.

> Brunet F., Kertudo P. et Malsan S., 2008, «Étude sociologique sur la résidence en alternance des enfants de parents séparés », Fors, Recherche sociale, Dossier d'étude n 109, Cnaf, octobre, p. 15.

$>$ Cadolle S., 2000, Être parent, être beau-parent. La recomposition de la famille, Paris, Odile Jacob ; 2008, « La résidence alternée : ce qu'en disent les mères », Informations sociales , $\mathrm{n}^{\circ} 149$, p. $68-81$. 
> Castelain-Meunier C., 2002, La place des hommes et les métamorphoses de la famille, Paris, Presses universitaires de France.

> Connell R. W., 1999, Masculinities, Cambridge, Polity Press.

$>$ Doucet A., 2006, Do Men Mother ? Fatherhood, Care and Domestic Responsability, Toronto, University of Toronto Press.

> Eydoux A. et Letablier M.-T., 2007, « Les familles monoparentales en France », rapport de recherche, Noisy-leGrand, Centre d'études de l'emploi.

> Jamoulle P., 2005, Des hommes sur le fil. La construction des identités masculines en milieu précaire, Paris, La Découverte.

> Martial A., 2003, S'apparenter. Ethnologie des liens de familles recomposées, Paris.

> Merla L., 2007, « Pères au foyer : une expérience hors norme », Recherches et prévisions, $\mathrm{n}^{\circ}$ 90, p. 23.

$>$ Neyrand G., 2000, L'enfant, la mère et la question du père. Un bilan critique de l'évolution des savoirs sur la petite enfance, Paris, Presses universitaires de France.

$>$ Regnier-Loilier A., 2006, « À quelle fréquence voit-on ses parents ? », Population et sociétés, $\mathrm{n}^{\circ} 427$, Ined.

$>$ Stewards S. D., 1999, «Disneyland Dads, Disneyland Mums ? How Nonresident Parents Spend Time with Absent Children », Journal of Family Issues, $n^{\circ} 20$, p. 539-556.

$>$ Villeneuve-Gokalp C., 1999, « La double famille des enfants de parents séparés $\gg$, Population, $n^{\circ} 1$, p. 9-36.

$>$ Vivas É., 2007, « La séparation des parents ou le décès de la mère distend les relations avec le père », Insee première, $n^{\circ} 1157$, Insee. 